\title{
Never say never
}

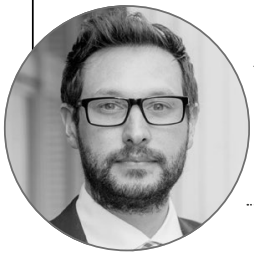

\section{David Westgarth}

Editor, BDJ in Practice

I $\mathrm{n}$ the coming years, countries, governments and healthcare systems around the world will look at the COVID-19 pandemic through the lens of a public inquiry. The World Health Organisation's team in Wuhan have already begun that process, looking at origins of the virus and if anything could have been done to prevent it, and if not, what can we do to prevent it from occurring in the future. It's something akin to the theory of 'Black Box Thinking' in the aviation sector - you only need to look at the events surrounding the Boeing 737 MAX to see the theory in action.

It is also a theory healthcare in the UK more often than not puts into practice. It has led to an environment where patient safety is first and foremost in any healthcare professional's mind as a result of extensive training, education and safety guidance/documents designed to ensure certain incidents they simply do not happen ever again.

Of course, I am talking about never events, which, after years of campaigning, finally contain no dental-specific events in England and Wales following the removal of wrong tooth extractions from the list. It marks a significant development, as it is now accepted that the national guidance in relation to tooth extraction does not provide the strong systemic barriers necessary to meet the definition of a never event, a change the BDA also successfully lobbied should be applied to wrong site blocks in 2019. However, a simple Google of 'what are never events in healthcare' took me down an Alice in Wonderland esq rabbit hole.

For starters, my top result for that search was to North Tees and Hartlepool NHS Foundation Trust document uploaded in 2015 that described never events as 'wholly preventable.' The second was to the CQC from 2018 that described them as 'largely preventable ${ }^{2}$ and the third to NHS England's latest update that described them as 'entirely preventable. ${ }^{3}$

So, which is it, and why the multiple descriptions? This journey through definitions of lexicon fascinates me. While 'wholly preventable' sounds absolute, one could argue that assessing multiple opinions and coming to a conclusion that 'on the whole' means there is some wiggle room, but not much. 'Largely preventable' is a definition that adds a significant dose of wiggle room, yet 'entirely preventable' closes it it $i s$ an absolute.

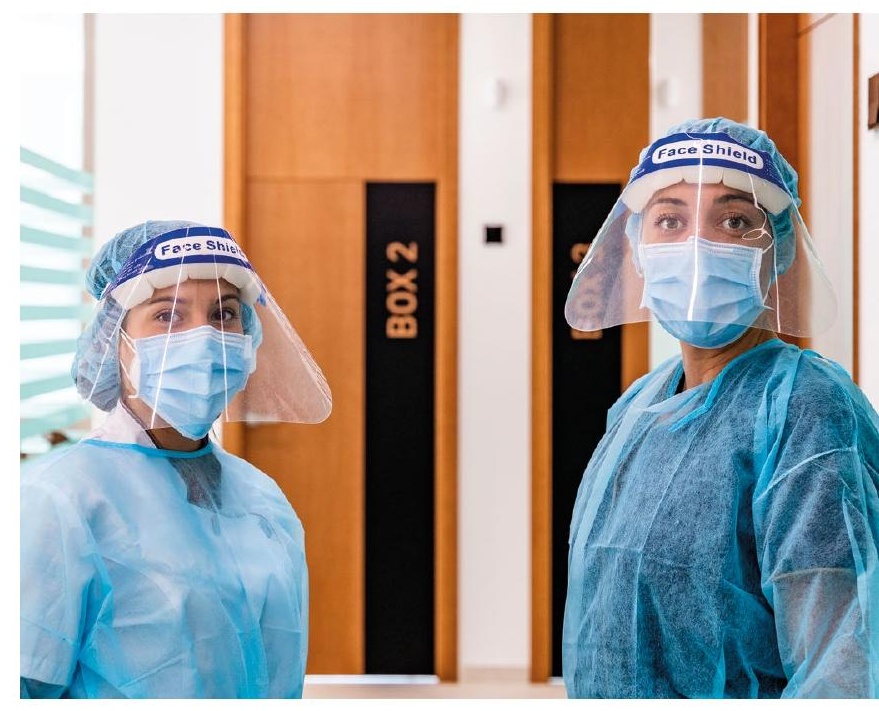

The change in definition over time certainly suggests dentistry has gone through its very own Black Box Thinking, tightening up 'loopholes' in terminology to make the profession a better place and assessing whether incidents meet the threshold for being placed on the list. How could you say never? As a child I had severe overcrowding and had teeth removed as a result. I am not alone in this - the NHS revision pointed to an example that 'patients often had different numbers and locations of teeth, e.g. as a result of previous dental work or due to their individual dental anatomy.'. I can only now imagine the poor dentist who took those out - counting, checking, double counting, double checking, triple counting, triple checking.

Fast forward to today, and extractions of decayed teeth in children, which take place in hospital under general anaesthetic, costing the NHS $£ 41.5$ million in $2018 / 19 .{ }^{4}$ More and more adults than ever before will have unique number and placement of teeth. The mental burden on dental professionals would have been immense. In fact, in 2019, then Chair of the GDPC, Dave Cottam, spoke about the 'terrible stigma' associated with never events. He said 'The stigma and fear surrounding a never-event is horrific for any dentist (as for any healthcare professional) to go through and was a completely disproportionate response to something that can occur even when a dentist follows the guidelines on wrong-site blocks to the letter' and that it was 'gratifying' that NHS England and NHS Improvement have agreed to a pragmatic and sensible change without compromising on patient safety.

Two years later, I am sure I speak for every dental professional when I say this is a further welcome development to the environment in which they operate within. The pandemic and its associated factors have been difficult enough for many, and while this may be a small scratch on the surface, it is one less burden to bear and puts an end to the unnecessary and highly stressful investigations associated with never-events in an already stressful practice, where you never have to say never again.

\section{References}

1. North Tees and Hartlepool NHS Foundation Trust. Never Events. Available online at: www. nth.nhs.uk/content/uploads/2015/05/neverevents.pdf (Accessed April 2021)

2. Care Quality Commission. Learning From Never Events. Available online at: www.cqc.org.uk/ news/stories/learning-never-events (Accessed April 2021).

3. NHS. Revised Never Events policy and framework. Available online at: www.england.nhs.uk/ patient-safety/revised-never-events-policy-andframework/ (Accessed April 2021).

4. Local Government Association. News release. 180 operations a day to remove rotten teeth in children. Available online at: www.local.gov.uk/ about/news/lga-nearly-180-operations-dayremove-rotten-teeth-children (Accessed April 2021).

https://doi.org/10.1038/s41404-021-0742-3 